\title{
Produção e qualidade do óleo essencial de menta em hidroponia com doses de potássio
}

\author{
Production and quality of essential oil of mint under hydroponics with four potassium levels
}

\author{
Tânea Maria Bisognin Garlet ${ }^{\mathrm{I}}$ Osmar Souza dos Santos ${ }^{\mathrm{II}}$ Sandro Luis Peter Medeiros ${ }^{\mathrm{II}}$ \\ Paulo Augusto Manfron ${ }^{\text {II }}$ Danton Camacho GarciaII Elis Borcioni ${ }^{\text {III }}$ Vinicius Fleig ${ }^{\mathrm{IV}}$
}

\section{RESUMO}

Este trabalho objetivou avaliar a produção de fitomassa de folhas, o teor e a qualidade do óleo essencial de Mentha $x$ gracilis Sole, cultivada em quatro doses de potássio nas soluções hidropônicas (276, 414, 552 e 690mg $L^{-1}$ ), no sistema "NFT" (Técnica do Fluxo Laminar de Nutrientes). Empregou-se delineamento experimental inteiramente casualizado, com cinco repetições. Estacas de plantas matrizes foram enraizadas em espuma fenólica, por 20 dias, em berçário, e, após isso, foram transferidas para bancadas de produção final. A colheita ocorreu aos 49 dias após o transplante. As folhas foram retiradas e pesadas para determinação da fitomassa fresca e aproveitadas para extração do óleo em aparelho de Clevenger. As análises de constituição química do óleo foram realizadas em Cromatógrafo Gasoso acoplado a Espectrômetro de Massa. As concentrações de K alteraram a produção de fitomassa fresca de folhas, o teor e a qualidade do óleo essencial. A dose máxima de K proporcionou aumento no teor do óleo essencial, porém reduziu a acumulação de fitomassa de folhas, diminuindo o rendimento do óleo por planta e a quantidade de linalol, o principal constituinte desse quimiotipo. A concentração de K sugerida para obtenção de maior rendimento da $\boldsymbol{M}$. $\boldsymbol{x}$ gracilis, em cultivo hidropônico, não deve ultrapassar $276 \mathrm{mg} L^{-1}$ na solução nutritiva.

Palavras-chave: Mentha x gracilis, Lamiaceae, linalol, óleo volátil.

\section{ABSTRACT}

This research was aimed at evaluating the production of leaves' phytomass, the content and quality of Mentha $x$ gracilis Sole essential oil with four potassium $(K)$ levels $\left(276,414,552\right.$ e 690mg $\left.L^{-1}\right)$ under hydroponics, on

\begin{abstract}
"NFT" system (Nutrient Film Technique). A completely randomized design was used with five replications. Stakes of matrix plants were rooted in phenolic foam for 20 days in a nursery, being then transferred to final production benches. Harvesting occurred at 49 days after transplanting. Leaves were separeted and weighted to determine fresch phytomass, and utilized for oil extraction in a Clevenger apparatus. The analyses of oil chemical constitution were performed in Gas Chromatography connected to a Mass Spectrometer. The K concentrations affected leaves fresh phytomass, content and quality of the essential oil. The K level maximum showed increase of essential oil yield, but decreased the fresch phytomass accumulation, decreasing the oil yield by plant and linalool quantity, the main constituent of this chemotype. The $K$ concentration suggested for the greatest yield of $M . x$ gracilis, under hydroponics, doesn't surpass $276 \mathrm{mg} \mathrm{L}^{-1}$ in nutritive solution.
\end{abstract}

Key words: Mentha x gracilis, Lamiaceae, linalool, volatile oil.

\section{INTRODUÇÃO}

Dentre as 25 espécies de Mentha (Lamiaceae), destaca-se Mentha x gracilis Sole, um complexo híbrido originado de $\boldsymbol{M}$. arvensis L. e $\boldsymbol{M}$. spicata L. (HARLEY \& BRIGHTON, 1977), que, através de uma série de cruzamentos, produz indivíduos estéreis e vários quimiotipos, todos com óleos essenciais ricos em monoterpenos. A composição química dos óleos essenciais pode variar conforme os

IPrograma de Pós-graduação em Agronomia (PPGA), Universidade Federal de Santa Maria (UFSM), Núcleo de Pesquisas em Ecofisiologia e Hidroponia (NUPECH), 97015-900, Santa Maria, RS, Brasil. E-mail: taneagarlet@hotmail.com. Autor para correspondência.

IIDepartamento de Fitotecnia, UFSM, NUPECH, Santa Maria, RS, Brasil.

IIIPPGA, UFSM, NUPECH, Santa Maria, RS, Brasil.

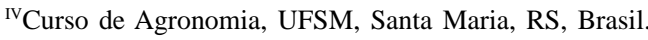


quimiotipos ou raças químicas que são determinados geneticamente e correspondem a vegetais botanicamente idênticos, mas que diferem quimicamente entre si. Para $\boldsymbol{M}$. x gracilis, foram caracterizados seis quimiotipos (TUCKER et al., 1991).

A produção mundial anual de óleos essenciais de trinta espécies aromáticas é estimada entre 110.000 e 120.000 toneladas (KHOTARI, 2005), e, desta quantidade, 22.200 toneladas vêm de espécies de Mentha: M. arvensis (16.000), M. x piperita (4.000), M. spicata (2.000) e outras (200) (SANT SANGANERIA, 2005). Esses óleos são amplamente usados na indústria de alimentos, medicamentos, aromatizantes e fragrâncias.

A biossíntese do óleo essencial ocorre em tricomas glandulares principalmente de folhas e cálices florais (LAWRENCE, 1992) e depende, além dos fatores genéticos, também dos fisiológicos e ambientais (FREITAS et al., 2004). As temperaturas ótimas ao crescimento de Mentha correspondem à noturna de $18^{\circ} \mathrm{C}$ e diurna de $30^{\circ} \mathrm{C}$, sendo que as diurnas exercem maior influência sobre o crescimento e rendimento do óleo essencial (DURIYAPRAPAN et al., 1986). Os nutrientes minerais também são fundamentais para o crescimento e produção de óleos (BROWN et al., 2003).

O potássio (K) é o nutriente mais abundante nos tecidos das plantas, não faz parte de nenhum composto orgânico, porém interfere em vários processos fisiológicos tais como: ativação enzimática de aproximadamente sessenta enzimas, síntese de amido e de proteínas, abertura e fechamento dos estômatos, fotossíntese, extensão celular, translocação de fotossintatos para partes em crescimento, resistência a doenças e melhor eficiência no uso da água (MARSCHNER, 1995; CAKMAK, 2005). Várias reações enzimáticas, nas plantas aromáticas, são necessárias para a síntese dos monoterpenos, que são formados através da rota do ácido mevalônico (CROTEAU et al., 2000).

Os nutrientes fornecidos às plantas podem ser controlados através das soluções nutritivas da hidroponia. A água enriquecida com nutrientes, aliada ao ambiente mais favorável obtido dentro das estufas, possibilita crescimento mais rápido, encurtando o ciclo produtivo e aumentando a produtividade (SANTOS, 2000). Além disso, o cultivo protegido e a ausência de solo reduzem muito o uso de defensivos agrícolas, oferecendo ao consumidor produtos de melhor qualidade. Desta forma, este trabalho teve como objetivo avaliar a produção de fitomassa de folhas, o teor e a qualidade do óleo essencial de Mentha x gracilis Sole, cultivada com quatro doses de potássio nas soluções hidropônicas.

\section{MATERIAL E MÉTODOS}

O experimento foi conduzido de outubro a dezembro de 2004, na Universidade Federal de Santa Maria, UFSM, RS, no Núcleo de Pesquisas em Ecofisiologia e Hidroponia (NUPECH), utilizando-se a técnica do fluxo laminar de nutrientes (NFT), em casa de vegetação do tipo arco pampeano, de $250 \mathrm{~m}^{2}$ de área, com filme plástico de polivinilclorídrico (PVC) de 200 $\mu \mathrm{m}$ de espessura para cobertura e fechamento das laterais e portas.

As mudas de $\boldsymbol{M}$. x gracilis foram produzidas a partir de matrizes cultivadas em solo, sendo obtidas a partir de estacas caulinares, de $4 \mathrm{~cm}$ de comprimento e quatro folhas, enraizadas em espuma fenólica por 20 dias em berçário, quando foram transplantadas para bancadas de produção final, cujo espaçamento dentro dos canais de cultivo foi de $0,25 \mathrm{~m}$ e, entre os canais, foi de $0,40 \mathrm{~m}$, correspondendo à densidade de 10 plantas $\mathrm{m}^{-2}$. Exsicata da espécie encontra-se depositada no Herbário da Universidade de Cruz Alta, RS (UNICRUZ), sob n. 1078, com determinação feita pelo Dr. Ray Harley, do Royal Botanic Gardens, Kew, Inglaterra.

O delineamento experimental utilizado foi o inteiramente casualizado, com cinco repetições. Foram estudadas quatro doses de K (tratamentos) na solução hidropônica (276, 414, 552 e 690 $\mathrm{mg} \mathrm{L}^{-1}$ ), utilizando-se a técnica do fluxo laminar de nutrientes (NFT). A solução nutritiva padrão de $\mathrm{K}\left(276 \mathrm{mg} \mathrm{L}^{-1}\right)$ foi calculada a partir de dados obtidos com $\boldsymbol{M}$. arvensis por PAULUS et al. (2004). Na produção de mudas, essa solução foi diluída a 50\%. Para seu preparo, utilizaram-se as seguintes doses de nutrientes $\left(\mathrm{mg} \mathrm{L}^{-1}\right)$ : $\mathrm{K}=276 ; \mathrm{N}=211,4 ; \mathrm{P}=30,35$; $\mathrm{Ca}=71,34 ; \mathrm{Mg}=32,56 ; \mathrm{S}=40,72 ; \mathrm{Mn}=6,084 ; \mathrm{Cu}=0,075$; $\mathrm{Zn}=0,431 ; \mathrm{B}=2,884 ; \mathrm{Mo}=0,088$. Foi utilizado 0,5L de FeEDTA obtido através da dissolução de $24,1 \mathrm{~g}$ de sulfato de ferro em 400mL de água e 25,1g de Na-EDTA em 400mL de água, misturando-se as duas soluções e completando-se o volume para 1,0L, efetuando-se, em seguida, borbulhamento de ar por $12 \mathrm{~h}$, no escuro, conforme recomendação de FURLANI \& FURLANI (1988). Os micronutrientes foram fornecidos na forma de solução concentrada (cinco vezes) dos sais em 200 $\mathrm{mL}$ de água, completando-se o volume para $1 \mathrm{~L}$, após completa diluição. A partir da solução padrão, variouse a concentração de K para 414, 552 e 690 $\mathrm{mg} \mathrm{L}^{-1}$ através da adição de 262,4; 524,8 e 787,2mg de $\mathrm{KCl} \mathrm{L}^{-1}$, respectivamente.

A condutividade elétrica (CE) inicial das soluções com 276, 414, 552 e $690 \mathrm{mg} \mathrm{L}^{-1}$ de $\mathrm{K}$ foi respectivamente 2,$38 ; 2,72 ; 3,30$ e $3,87 \mathrm{mS} \mathrm{cm}^{-1}$. O controle foi realizado a cada dois dias, sendo feita a reposição de $50 \%$ dos nutrientes sempre que a CE 
atingia $50 \%$ do valor inicial. $\mathrm{O} \mathrm{pH}$ foi corrigido a cada dois dias para o valor de 6,0 0 ,2, após completar-se o volume dos reservatórios com água. O controle da circulação das soluções foi efetuado com auxílio de temporizador programado para acionar a moto-bomba durante 15 minutos, com intervalos de 15 minutos, no período diurno (07:00-20:00h), e 15 minutos a cada intervalo de $2 \mathrm{~h}$ no período noturno. Os dados de temperatura do ar foram obtidos a partir de termohigrógrafo, instalado a 1,5m de altura do piso da casa de vegetação.

A colheita das plantas foi realizada aos 49 dias após o transplante, em dezembro de 2004, quando as plantas estavam em início de floração, para melhor aproveitamento do óleo essencial. As folhas foram retiradas e pesadas para determinação de fitomassa fresca, sendo após acondicionadas em sacos plásticos e mantidas em freezer até o momento da extração do óleo. Amostras de $100 \mathrm{~g}$ de folhas, em quatro repetições, foram usadas para hidrodestilação em aparelho de Clevenger por $2 \mathrm{~h}$. O óleo essencial obtido foi separado da água e secado com $\mathrm{Na}_{2} \mathrm{SO}_{4}$, sendo pesado para determinação do teor e rendimento estimados por planta e por hectare.

As análises de constituição química do óleo essencial foram realizadas em Cromatógrafo Gasoso acoplado a Espectrômetro de Massa (CG-EM Shimadzu, QP-5000), da Faculdade de Farmácia da Universidade Federal do Rio Grande do Sul, UFRGS. A identificação dos constituintes foi efetuada por comparação de seus respectivos espectros de massas e índices de retenção com amostras autênticas e dados obtidos na literatura (ADAMS, 2001) e ainda por comparação com espectros de massas registrados em banco de dados como NIST 62 e NIST 12 (National Institute of Standars and Technology).

Os dados obtidos foram submetidos à análise de variância e interpretados por meio de análise de regressão, utilizando-se teste $\mathrm{F}$ a 1 e $5 \%$ de probabilidade para avaliação das equações polinomiais.

\section{RESULTADOS E DISCUSSÃO}

As concentrações de potássio influenciaram significativamente $(\mathrm{P}<0,01)$ a produção de fitomassa fresca de folhas (g) e o teor de óleo essencial em Mentha x gracilis Sole, colhida aos 49 dias após o transplante (Figura 1). A dose estimada de K para obtenção da melhor produtividade de folhas frescas corresponde à primeira dose utilizada de $276 \mathrm{mg} \mathrm{L}^{-1}$, pois a aplicação de mais do que $276 \mathrm{mg} \mathrm{L}^{-1}$ de $\mathrm{K}$ prejudicou a produção de fitomassa. O teor de óleo essencial aumentou com o acréscimo de K na solução hidropônica, obtendo-se
1,19 g de óleo para cada $100 \mathrm{~g}$ de folhas frescas na dose de $690 \mathrm{mg} \mathrm{L}^{-1}$. Assim, com menor produção de fitomassa, houve aumento na concentração de óleo.

Embora o teor do óleo essencial (\%) tenha aumentado na maior dose de $\mathrm{K}$, esta ocasionou o menor rendimento, que foi de 2,56g de óleo planta ${ }^{-1}$. De acordo com DURIYAPRAPAN et al. (1986), o conteúdo de óleo em Mentha tende a incrementar com o aumento da temperatura de 30 para $35^{\circ} \mathrm{C}$, acompanhado da redução no crescimento das plantas. Durante o período experimental, a temperatura no interior da casa de vegetação foi favorável ao crescimento das plantas de M. x gracilis, cujos valores observados situaram-se próximos à faixa de $18 \mathrm{a} 34^{\circ} \mathrm{C}$. Os resultados do presente estudo concordam com DURIYAPRAPAN et al. (1986) e também com TUOMI et al. (1991), que afirmam que a concentração de metabólitos secundários utilizados para defesa das plantas tende a apresentar concentração inversa às taxas de crescimento, havendo então desvio de compostos do metabolismo primário (açúcares, proteínas, lipídios) para produção de metabólitos secundários, tais como os terpenóides.

A ampla utilização de óleos essenciais e seus derivados tem motivado alguns estudos agronômicos de espécies de Mentha. TELCI et al. (2004) obtiveram conteúdo médio de $1,66 \%$ de óleo de $\boldsymbol{M}$. spicata cultivada a campo, com variações entre 0,90 e 2,70\%, sendo que o conteúdo do óleo foi influenciado por condições ambientais de temperatura e intensidade luminosa; temperaturas entre 21,1 e $22,3^{\circ} \mathrm{C}$ proporcionaram plantas com alto conteúdo em óleo.

Em cultivo hidropônico de $\boldsymbol{M}$. arvensis, PAULUS et al. (2004) conseguiram teor de $0,60 \%$ de óleo essencial, em solução nutritiva contendo $299 \mathrm{mg}$ $\mathrm{L}^{-1}$ de K, enquanto MAIA et al. (2001) obtiveram o maior teor de $1,45 \%$, em solução com $468 \mathrm{mg} \mathrm{L}^{-1}$ de $\mathrm{K}$, evidenciando que as soluções nutritivas para a espécie devem ser mais concentradas em K. Entretanto, VALMORBIDA(2003) encontrou teor médio de 1,33\% de óleo essencial obtido de plantas de $\boldsymbol{M}$. x piperita, sem que houvesse efeito significativo com a variação nos diferentes níveis de K nas soluções nutritivas. Em cultivo em solo de $\boldsymbol{M}$. x piperita, PRAZNA \& BERNÁTH (1993) constataram decréscimo no conteúdo do óleo essencial em plantas cultivadas na ausência de $\mathrm{K}$; no entanto, o pós-tratamento com $\mathrm{K}$ a essas plantas indicou haver aumento na quantidade do óleo essencial.

Em experimentos a campo com $\boldsymbol{M}$. arvensis, a colheita de plantas frescas permitiu, segundo RAPJUT et al. (2002), rendimento de fitomassa total de 13 ha $^{-1}$ e de óleo essencial de 71,5kg ha ${ }^{-1}$. SRIVASTAVA et al. (2002) conseguiram fitomassa de folhas de 16,5 


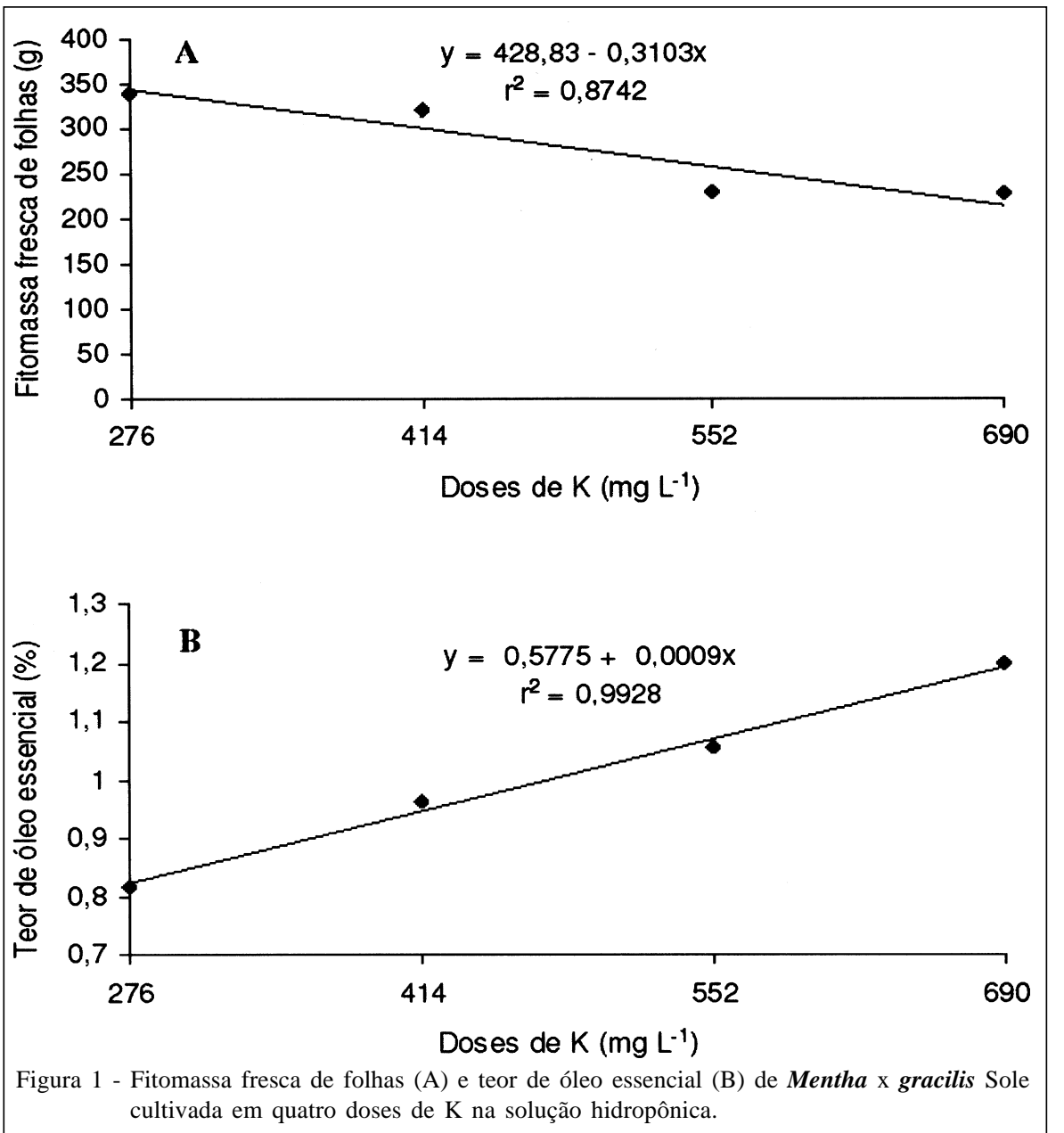

toneladas ha-1 e 114,0kg de óleo essencial hä ${ }^{-1}$. Para $\boldsymbol{M}$. spicata, TELCI et al. (2004) obtiveram rendimento médio de fitomassa de folhas de 16,6 toneladas ha $^{-1}$ e de óleo entre 275,7 e $320,5 \mathrm{~kg} \mathrm{ha}^{-1}$. Neste estudo, o máximo rendimento obtido de fitomassa de folhas foi equivalente a 34,3 toneladas ha-1 e de óleo essencial de $285 \mathrm{~kg} \mathrm{ha}^{-1}$, indicando ótima produtividade em hidroponia.

A destilação das folhas de $\boldsymbol{M}$. x gracilis resultou em óleos essenciais de coloração levemente amarelada e suave odor de lavanda, e, através das análises cromatográficas, revelou a identificação de 23 constituintes, que na média correspondem a 98,13\% do total. Observando-se os dados da tabela 1, verificam-se diferenças quantitativas $(\mathrm{P}<0,01$ e $\mathrm{P}<0,05)$ na composição química das amostras de óleo examinadas. Os componentes majoritários encontrados foram linalol (39,90-49,50\%), carvona (11,10-13,90\%) e cis-hidrato de sabineno (10,50-12,41\%). Outros componentes que apresentaram significância foram alfa-pineno (0,10-0,32\%), beta-pineno (0,23-0,63\%), mirceno (0,53-1,60\%), limoneno (1,20-2,53\%), terpinoleno (0,10-0,30\%), alfa-terpineol (0,53-0,90\%) e pulegona (0,10-0,65\%).

O conteúdo de linalol e pulegona no óleo foi menor nas plantas submetidas à maior concentração de $\mathrm{K}$, conforme constatado pelas equações de regressão da tabela 2. No entanto, para os componentes alfa-pineno, beta-pineno, mirceno, limoneno, cis-hidrato de sabineno, terpinoleno, alfa-terpineol e carvona, conforme aumentou a concentração de K, houve também incremento nos seus conteúdos. Tais resultados sugerem que o $\mathrm{K}$ tenha afetado a atividade de enzimas responsáveis pela biossíntese desses componentes do óleo essencial de $\boldsymbol{M}$. x gracilis.

MAIA et al. (2001) citam que a disponibilidade de nutrientes no meio pode induzir a atividade enzimática para os constituintes do óleo e, no estudo com Mentha arvensis, aumentar o teor de mentol e a qualidade do óleo essencial. Os autores obtiveram o maior conteúdo de mentol (82,70\%) na solução mais concentrada em $\mathrm{K}$ com $468 \mathrm{mg} \mathrm{L}^{-1}$. Todavia, VALMORBIDA (2003), testando a solução n. 2 de 
Tabela 1 - Composição química do óleo essencial (\%) obtida em CG-EM, de Mentha x gracilis Sole cultivada em solução nutritiva com diferentes doses de $\mathrm{K}$, colhida aos 69 dias após o plantio. ${ }^{1} \mathrm{TR}=$ tempo de retenção médio; ${ }^{2} \mathrm{IK}=$ Índice de Kovats; tr= traços $(<0,1 \%)$.

\begin{tabular}{|c|c|c|c|c|c|c|c|}
\hline \multirow{2}{*}{ Componente (\%) } & \multicolumn{5}{|c|}{ Dose de $\mathrm{K}\left(\mathrm{mg} \mathrm{L}^{-1}\right)$} & \multirow{2}{*}{${ }^{1} \mathrm{TR}$ (min.) } & \multirow{2}{*}{${ }^{2} \mathrm{IK}$} \\
\hline & 276 & 414 & 552 & 690 & Média & & \\
\hline Alfa-pineno** & 0,10 & 0,17 & 0,29 & 0,32 & 0,22 & 5,192 & 923 \\
\hline Sabinenons $^{\text {ns }}$ & 0,40 & 0,40 & 0,80 & 0,77 & 0,59 & 5,804 & 961 \\
\hline Beta-pineno* & 0,23 & 0,40 & 0,59 & 0,63 & 0,46 & 5,870 & 964 \\
\hline 3-octanona ${ }^{\text {ns }}$ & 2,93 & 2,20 & 3,43 & 4,31 & 3,22 & 5,964 & 978 \\
\hline Mirceno* & 0,53 & 1,07 & 1,43 & 1,60 & 1,16 & 6,044 & 980 \\
\hline 3 -octanol ${ }^{\text {ns }}$ & 10,63 & 11,73 & 11,61 & 11,50 & 11,37 & 6,152 & 985 \\
\hline Alfa-terpineno ${ }^{\mathrm{ns}}$ & 0,20 & 0,17 & 0,25 & 0,29 & 0,23 & 6,453 & 1009 \\
\hline Limoneno* & 1,20 & 1,80 & 2,37 & 2,53 & 1,97 & 6,636 & 1021 \\
\hline $1,8-$ cineol $^{\mathrm{ns}}$ & 2,33 & 2,10 & 3,20 & 2,77 & 2,60 & 6,681 & 1024 \\
\hline (Z)-beta-ocimeno ${ }^{\text {ns }}$ & 0,30 & 0,33 & 0,40 & 0,55 & 0,39 & 6,718 & 1031 \\
\hline (E)-beta-ocimeno ${ }^{\mathrm{ns}}$ & 0,17 & 0,17 & 0,27 & 0,27 & 0,22 & 6,869 & 1039 \\
\hline Gama-terpineno $^{\text {ns }}$ & 0,37 & 0,30 & 0,53 & 0,53 & 0,43 & 7,044 & 1050 \\
\hline Cis-hidrato de sabineno** & 10,50 & 11,90 & 13,08 & 14,17 & 12,41 & 7,224 & 1059 \\
\hline Terpinoleno* & 0,10 & 0,17 & 0,26 & 0,30 & 0,21 & 7,458 & 1085 \\
\hline Linalol* & 49,50 & 47,37 & 42,34 & 39,90 & 44,77 & 7,690 & 1093 \\
\hline Terpinen-4-ol $^{\text {ns }}$ & 1,77 & 1,53 & 1,53 & 1,75 & 1,65 & 8,615 & 1173 \\
\hline Alfa-terpineol* & 0,53 & 0,63 & 0,80 & 0,90 & 0,72 & 8,766 & 1178 \\
\hline Trans-diidrocarvona & $\operatorname{tr}$ & $\operatorname{tr}$ & $\operatorname{tr}$ & - & - & 8,836 & 1198 \\
\hline Pulegona* & 0,57 & 0,65 & 0,43 & 0,10 & 0,44 & 9,357 & 1224 \\
\hline Carvona** & 11,10 & 12,27 & 13,20 & 13,90 & 12,62 & 9,410 & 1236 \\
\hline Beta-cariofileno $^{\text {ns }}$ & 1,53 & 1,73 & 1,80 & 1,87 & 1,73 & 11,342 & 1408 \\
\hline Alfa-humuleno ${ }^{\mathrm{ns}}$ & 0,13 & 0,13 & 0,10 & 0,14 & 0,13 & 11,578 & 1439 \\
\hline Germacreno D ${ }^{\mathrm{ns}}$ & 0,43 & 0,57 & 0,70 & 0,69 & 0,59 & 11,827 & 1469 \\
\hline Total identificado & 95,55 & 97,79 & 99,41 & 99,79 & 98,13 & & \\
\hline
\end{tabular}

${ }^{* *} \mathrm{e}^{*}=$ significativo a $1 \%$ e $5 \%$ de probabilidade de erro pelo teste $\mathrm{F}$, respectivamente; ${ }^{\text {ns }}=$ não significativo.

Hoagland e Arnon e variando o nível de K pela redução de 50 e $75 \%$ da concentração, não observou efeito sobre o teor e a constituição química do óleo essencial de $\boldsymbol{M}$. x piperita.
A rota da biossíntese dos monoterpenos em Mentha x piperita está bem estabelecida (CROTEAU et al., 2000), e a produção dos monoterpenos está restrita ao desenvolvimento de glândulas de óleo de

Tabela 2 - Equações de regressão, coeficientes de determinação $\left(\mathrm{R}^{2}\right)$ e coeficientes de variação (CV) para os componentes do óleo essencial de Mentha x gracilis Sole que apresentaram significância pelo teste F.

\begin{tabular}{llll}
\hline Componente (\%) & \multicolumn{1}{c}{ Equação } & $\mathrm{R}^{2}$ & $\mathrm{CV}(\%)$ \\
\hline Alfa-pineno & $\mathrm{y}=-0,052+0,00056 \mathrm{x}$ & $0,9499^{* *}$ & 27,54 \\
Beta-pineno & $\mathrm{y}=-0,023+0,00101 \mathrm{x}$ & $0,9418^{* *}$ & 20,56 \\
Mirceno & $\mathrm{y}=-0,090+0,00258 \mathrm{x}$ & $0,9497 * *$ & 16,72 \\
Limoneno & $\mathrm{y}=0,377+0,00331 \mathrm{x}$ & $0,9511^{*}$ & 19,56 \\
Cis-hidrato de sabineno & $\mathrm{y}=8,148+0,00883 \mathrm{x}$ & $0,9965^{* *}$ & 5,70 \\
Terpinoleno & $\mathrm{y}=-0,039+0,00051 \mathrm{x}$ & $0,9829 *$ & 30,64 \\
Linalol & $\mathrm{y}=56,616-0,02451 \mathrm{x}$ & $0,9744^{*}$ & 8,362 \\
Alfa-terpineol & $\mathrm{y}=0,273+0,00092 \mathrm{x}$ & $0,9890^{*}$ & 18,01 \\
Pulegona & $\mathrm{y}=1,005-0,00056 \mathrm{x}$ & $0,7406^{*}$ & 38,38 \\
Carvona & $\mathrm{y}=9,350+0,00676 \mathrm{x}$ & $0,9877 * *$ & 5,79 \\
\hline
\end{tabular}

${ }^{* *} \mathrm{e}^{*}=$ significativo a $1 \%$ e $5 \%$ de probabilidade de erro pelo teste $\mathrm{F}$, respectivamente.

Ciência Rural, v.37, n.4, jul-ago, 2007. 
folhas jovens (MAHMOUD et al., 2004). Recentes evidências indicam que o principal determinante na produção de monoterpenos em Mentha seja a taxa de biossíntese, definida pelos níveis regulados de desenvolvimento das enzimas biossintéticas responsáveis e seus substratos correspondentes (TURNER et al., 2000). Isso foi constatado por GERSHENZON et al. (2000), que verificaram a existência de correlação entre as atividades in vitro e in vivo para oito enzimas da biossíntese do mentol.

O geranil-difosfato é o primeiro composto precursor de outros monoterpenos em espécies de Mentha (LINCOLN et al., 1986; CROTEAU et al., 2000; MAHMOUD et al., 2004), conforme se observa na figura 2, e pode originar, através de várias reações enzimáticas, 1,8-cineol, sabineno, hidrato de sabineno, linalol, acetato de linalila e limoneno. O limoneno é o precursor da carvona e, por reações sucessivas a partir da pulegona, pode formar mentona, mentol, isomentona, neoisomentol e/ou mentofurano. A interconversão dos componentes de um a outro é determinada geneticamente e pode ser afetada por fatores agronômicos (MAIA et al., 2001; TELCI et al., 2004; SCAVRONI et al., 2005). Com base nisso, as condições de variação nas concentrações de $K$ nas soluções hidropônicas às quais as plantas de $\boldsymbol{M}$. $\mathrm{x}$ gracilis foram submetidas favoreceram a formação dos compostos linalol, carvona e cis-hidrato de sabineno.

As várias aplicações dos constituintes dos óleos das mentas pela indústria justificam o interesse pelos monoterpenos. Segundo THE MERCK INDEX (1996), o linalol é empregado em perfumaria em substituição aos óleos de bergamota (mexerica) e de lavanda. A carvona, conforme CARVALHO \& FONSECA (2006), é importante agente antimicrobiano contra bactérias e fungos patogênicos, daí o seu emprego como composto isolado ou através do óleo, em alimentos e produtos anti-sépticos. Possui também atividade inseticida, atuando contra moscas das frutas, larvas de insetos, inclusive sobre Aedes aegypti, o vetor da dengue hemorrágica.

As folhas, sendo o sítio de síntese e acumulação do óleo, constituem-se de grande relevância para aumento do conteúdo e dos constituintes do óleo essencial de interesse para a indústria. Vários esforços têm sido feitos para aumentar o rendimento de monoterpenos em plantas, a fim de responder à demanda crescente mundial por aromatizantes, flavorizantes, fragrâncias e medicamentos. Considerando os resultados aqui obtidos, sugere-se que o potássio deva estar envolvido na síntese de compostos aromáticos, pois, segundo MARSCHNER (1995), é ativador de várias enzimas, destacando-se as sintetases, oxirredutases, desidrogenases, transferases, quinases e aldolases. As condições de aumento na concentração desse nutriente podem ter provocado estimulação nas atividades enzimáticas, alterando conseqüentemente a composição dos óleos.

\section{CONCLUSÃO}

As concentrações de $K$ nas soluções hidropônicas alteram a produção de fitomassa fresca de folhas, o teor e a qualidade do óleo essencial de Mentha x gracilis Sole. A dose máxima de K $\left(690 \mathrm{mg} \mathrm{L}^{-1}\right)$ proporciona aumento no teor do óleo essencial, porém reduz a acumulação de fitomassa de folhas, diminuindo o rendimento do óleo por planta e a quantidade de linalol, o principal constituinte deste quimiotipo. A concentração de $\mathrm{K}$ sugerida para obtenção de maior rendimento de $\boldsymbol{M}$. x gracilis, em cultivo hidropônico, nas condições ambientais do presente trabalho, não deve ultrapassar $276 \mathrm{mg} \mathrm{L}^{-1}$ na solução nutritiva.

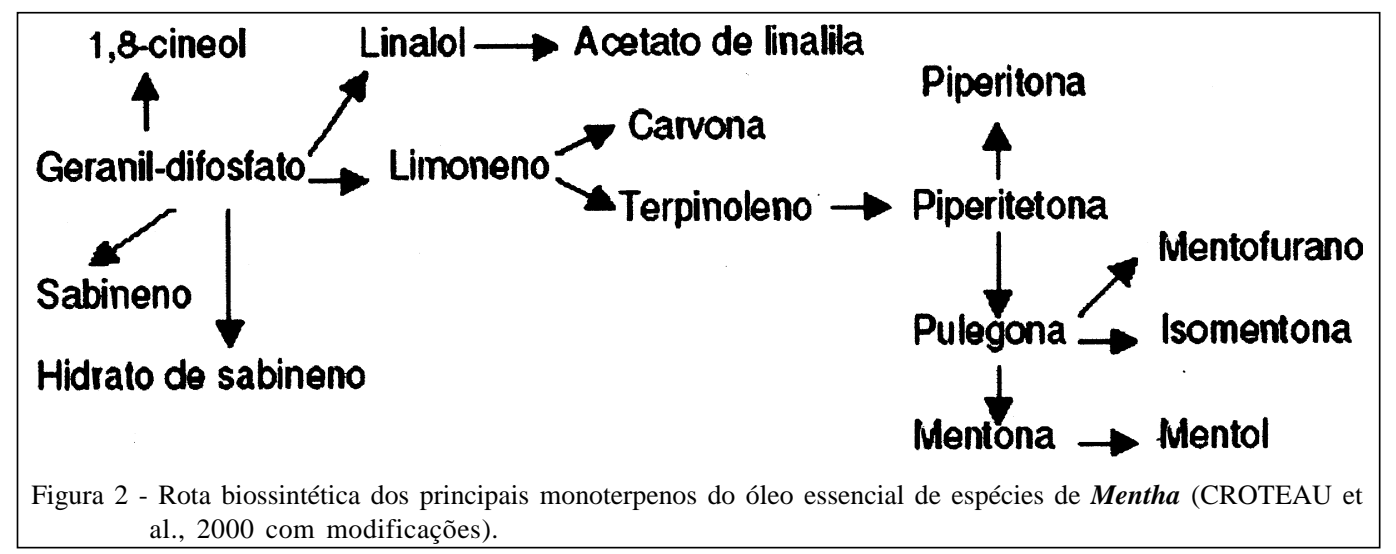

Ciência Rural, v.37, n.4, jul-ago, 2007. 


\section{REFERÊNCIAS}

ADAMS, R.P. Identification of essencial oils components by gas cromatography/ quadrupole mass spectroscopy. Illinois: Allured Publ. Corp. Carol Stream, 2001. 469p.

BROWN, B. et al. The critical role of nutrient management in mint production. Better Crops, v.87, n.4, p.9-11, 2003.

CAKMAK, I. Protection of plants from detrimental effects of enviromenmental stress factors. In: YAMADA, T; ROBERTS, T.L. In: SIMPÓSIO SOBRE POTÁSSIO NA AGRICULTURA BRASILEIRA, 2., 2004, São Pedro, SP. Anais... Piracicaba: Associação Brasileira para Pesquisa da Potassa e do Fosfato, 2005. p.261-279.

CARVALHO, C.C.R.; FONSECA, M.M.R. Carvone: why and how should one bother to produce this terpene. Food Chemistry, v.95, p.413-422, 2006.

CROTEAU, R. et al. Natural products (secondary metabolites). In: BUCHANAM, B.B. et al. Biochemistry \& molecular biology of plants. Rockville: Courier Companies, 2000. p.1250-1318.

DURIYAPRAPAN, S. et al. The effect of temperature on growth, oil yeld and oil quality of Japanese mint. Annals of Botany, v.58, n.5, p.729-736, 1986.

FREITAS, M.S. et al. Produção e qualidade de óleos essenciais de Mentha arvensis em resposta à inoculação de fungos micorrízicos arbusculares. Pesquisa Agropecuária Brasileira, v.39, n.9, p.887-894, 2004.

FURLANI, A.M.C.; FURLANI, P.R. Composição e pH de soluções nutritivas para estudos fisiológicos e seleção de plantas em condições nutricionais adversas. Campinas: Instituto Agronômico, 1988. 34p.

GERSHENZON, J. et al. Regulation of monoterpene accumulation in leaves of peppermint. Plant Physiology, v.122, p.205-213, 2000.

HARLEY, R.M.; BRIGHTON, C.A. Chromosome numbers in the genus Mentha $L$. Botanical Journal of the Linnean Society, v.74, p.71-96, 1977.

KOTHARI, R. The indian essential oil industry. Perfumer and flavorist, v.30, p.46-50, 2005.

LAWRENCE, B.M. Chemical components of Labiatae oils and their exploitation. In: HARLEY, R.M.; REYNOLDS, T. (Eds). Advances in Labiatae science. Kew: Royal Botanic Gardens, 1992. p.399-436.

LINCOLN, D. et al. Chemical composition and genetic basis for the isopinocamphone chemotype of Mentha citrata hybrids. Phytochemistry, v.25, n.8, p.1857-1863, 1986.

MAHMOUD, S.S. et al. Cosuppresion of limonene-3hydroxylase in peppermint promotes accumulation of limonene in the essential oil. Phytochemistry, v.65, p.547-554, 2004.
MAIA, N.B. et al. Essential oil production and quality of Mentha arvensis L. grower in nutrient solutions. Acta Horticulturae, n.548, p.181-187, 2001.

MARSCHNER, H. Mineral nutrition of higher plants. San Diego: Academic, 1995. 888p.

PAULUS, D. et al. Rendimento de biomassa e óleo essencial de menta japonesa (Mentha arvensis L.). Revista Brasileira de Plantas Medicinais, v.7, n.1, p.34-42, 2004.

PRAZNA, F.; BERNÁTH, J. Correlations between the limited level of nutrition and essential oil production of peppermint. Acta Horticulturae, v.344, p.278-289, 1993.

RAPJUT, D.K. et al. Response of cornmint (Mentha arvensis L. f. piperascens Malinv. ex Holmes) to micronutrients. Journal of Horticultural Science \& Biotechnology, v.77, n.4, p.438-440, 2002.

SANT SANGANERIA. Vibrant India. Opportunities for the flavor and fragrance industry. Perfumer and flavorist, v.30, p.24-34, 2005

SANTOS, O.S. (Ed). Cultivo sem solo: hidroponia. Santa Maria: UFSM/CCR, 2000. 107p.

SCAVRONI, J. et al. Yield and composition of the essential oil of Mentha piperita L. (Lamiaceae) grown with biosolid. Brazilian Journal of Plant Physiology, v.17, n.4, p.345352, 2005.

SRIVASTAVA, R.K. et al. Characteristics of menthol mint Mentha arvensis cultivated on industrial scale in the IndoGangetic plains. Industrial Crops and Products, v.15, p.189198, 2002.

TELCI, I. et al. Agronomical and chemical characterization of spearmint (Mentha spicata L.) originating in Turkey. Economic Botany, v.58, n.4, p.721-728, 2004.

THE MERCK INDEX. 12.ed. Whitehouse Station, NJ: Merck, 1996. p.939.

TUCKER, A.O. et al. The origin of Mentha x gracilis (Lamiaceae). II. Essential oils. Economic Botany, v.45, n.2, p.200-215, 1991.

TUOMI, J. et al. Carbon allocation, phenotypic plasticity and induced defences. In: TALLAMY, D.W.; RAUPP, M.J. Phytochemical induction by herbivores. New York: John Wiley, 1991. p.85-104.

TURNER, G.W. et al. Distribution of peltate glandular trichomes on developing leaves of peppermint. Plant Physiology, v.124, p.655-663, 2000.

VALMORBIDA, J. Níveis de potássio em solução nutritiva, desenvolvimento de plantas e a produção de óleo essencial de Mentha piperita L. 2003. 28f. Dissertação (Mestrado em Agronomia/ Horticultura)- Faculdade de Ciências Agronômicas, Universidade Estadual Paulista, Botucatu. 